\title{
EFECTOS DE LA ESCOLARIDAD EN EL CONSUMO DE CALORÍAS Y NUTRIENTES DE LAS FAMILIAS MEXICANAS
}

\section{Owen Eli Ceballos Mina y Humberto Guadarrama Gómez}

Fecha de recepción: 5 de febrero de 2020. Fecha de aceptación: 6 de julio de 2020.

$$
\text { http://doi.org/10.22201/iiec.20078951e.2020.203.69569 }
$$

Resumen. Este artículo describe el consumo de calorías y micronutrientes sobre la
distribución de riqueza y los niveles de escolaridad en los hogares mexicanos. Empleando
datos de la eNIGH-2018 se estiman regresiones semiparamétricas del consumo calórico,
así como de cinco micronutrientes a lo largo de la distribución de gastos y de los años
de educación del jefe de hogar. A mayor nivel económico se registra más consumo de
calorías y de micronutrientes; cuando se controla por la escolaridad, los hogares con
menor capital humano realizan consumos energéticos elevados en detrimento de la
ingesta de nutrientes. Se revela también que conforme la escolaridad se incrementa, los
hogares consumen cantidades calóricas cercanas a las recomendadas y tienen preferencias
por alimentos más ricos en micronutrientes.

Palabras clave: consumo de calorías; nutrición; escolaridad; hogares; nivel económico; regresiones semiparamétricas.

Clasificación JEL: C14; D12; I12; L66.

\section{Effects of Education on Calorie and Nutrient Intake in Mexican Families}

\begin{abstract}
This article analyzes calorie and micronutrient intake in relation to wealth distribution and education levels in Mexican households. Using data from the National Survey on Income and Household Expenditure, 2018 (ENIGH-2018), semiparametric regressions of intake of calories and five micronutrients are estimated across the distribution of expenditure and the head of household's education level. Higher economic status households consumed more calories and micronutrients; when controlled according to level of education, however, households with lower human capital demonstrated an increased caloric intake while simultaneously demonstrating a reduced nutrient intake. Findings also showed that as education level increases, households consume closer to recommended caloric intakes and show a preference for more micronutrient-dense foods.
\end{abstract}

Key Words: calorie intake; nutrition; education; households; economic status; semiparametric regressions.

\footnotetext{
a Universidad Autónoma Metropolitana, Unidad Azcapotzalco, México. Correos electrónicos: oecm@azc.uam.mx y humb.gg@gmail.com, respectivamente.
} 


\section{INTRODUCCIÓN}

Durante las últimas décadas, la malnutrición es cada vez un problema más complejo y de alcance mundial (oms, 2018). Si bien en América Latina la desnutrición solía ser un fenómeno extendido en la región, hoy en día se observa lo que la Comisión para América Latina y el Caribe (CEPAL) define como la doble carga de la malnutrición; esto es, la coexistencia de malnutrición tanto por déficit como por exceso de calorías y nutrientes (Fernández et al., 2017). Aunque existen varias explicaciones que dan cuenta de los cambios en el perfil nutricional de los mexicanos (Torres, 2017), el tema no es de menor importancia, pues ya desde el 2000 los índices de sobrepeso y obesidad se han incrementado drásticamente (Rivera et al., 2017), mientras la desnutrición todavía afecta a casi $24 \%$ de la población (CONEVAL, 2015).

Buena parte de los estudios que estiman la ingesta de calorías y nutrientes sugieren que la capacidad financiera de las familias es uno de los principales determinantes del consumo alimenticio (Drewnowski y Darmon, 2005; Drewnowski y Specter, 2004; Hernández et al., 2017). Aunque dichos estudios han obtenido resultados relevantes sobre la importancia del ingreso para analizar disparidades en el consumo de calorías, existe poca claridad respecto a los efectos del nivel socioeconómico sobre la ingesta de micronutrientes. Estos análisis tampoco incluyen otros controles sociodemográficos que pueden incidir en la alimentación de las familias. Por ejemplo, la escolaridad es una variable que no sólo informa sobre la acumulación de capital humano, sino que es indicador de muchas de las capacidades presentes en el seno familiar.

El objetivo de este trabajo es caracterizar el perfil de consumo de calorías y de micronutrientes a lo largo de la distribución socioeconómica de los hogares mexicanos y estimar los efectos de la escolaridad sobre el mencionado consumo. Para ello, se emplean datos de las cantidades de alimento consumidos dentro y fuera del hogar contenidas en la Encuesta Nacional de Ingresos y Gastos de los Hogares (ENIGH) de 2018. A diferencia de otros estudios que se centran en el consumo de energía, el presente trabajo incluye información adicional sobre hierro, zinc, calcio, vitamina A y vitamina C. El estudio parte de la transformación de cantidades de alimentos en consumo calórico y de micronutrientes para estimar regresiones no paramétricas y semiparamétricas de dichos consumos sobre la distribución de gasto y sobre los años de educación del jefe de familia, controlando por un conjunto de características sociodemográficas de los hogares.

Los resultados muestran que, a mayor nivel socioeconómico y de gasto se tiene un mayor consumo de calorías y micronutrientes en los hogares; sin 
embargo, cuando se controla por el nivel de escolaridad, se observa que en los hogares con baja o nula escolaridad del jefe de familia se tiende a realizar un consumo energético elevado por encima de los estándares recomendados sacrificando el consumo de micronutrientes. Conforme la escolaridad se incrementa, los hogares consumen cantidades calóricas cercanas a las recomendadas por diversos organismos de salud, y muestran una mayor preferencia por alimentos ricos en micronutrientes. Dichos hallazgos coinciden con otros trabajos aplicados al caso de México, como los de Hernández et al. (2017), Pérez y Minor (2011) y Martínez y Villezca (2005).

Este artículo se estructura en cinco secciones incluyendo esta introducción, en la segunda sección se presenta una revisión de la literatura sobre nutrición, nivel económico y escolaridad; en la tercera sección se desarrolla la estrategia de identificación, se inicia una descripción del perfil nutricional adultoequivalente de las familias con la ENIGH-2018, se presentan además modelos usados para el análisis de los efectos de la escolaridad sobre el consumo de calorías y micronutrientes; en la cuarta sección, se exponen los resultados de las estimaciones de los determinantes del consumo de calorías y micronutrientes; en la quinta sección, a manera de conclusión, se discuten las implicaciones de las desigualdades educativas y nutricionales de los hogares en México.

\section{ESTUDIOS SOBRE NUTRICIÓN, NIVEL ECONÓMICO Y ESCOLARIDAD}

La nutrición es uno de los factores con mayor influencia en los resultados de vida de las personas; el estado nutricional posee efectos sobre el aprendizaje y la productividad de los individuos, por lo que el análisis de su comportamiento es de gran interés para el crecimiento y desarrollo económico. El consumo energético y de nutrientes se considera en la literatura económica como una inversión en capital humano (Schultz, 1961 y 1962); sin embargo, enfoques más recientes como el de capacidades, tratan los aspectos nutricionales como un elemento estratégico de la forma en que las personas pueden alcanzar estados del ser y realizar actividades valiosas (Nussbaum, 2011).

En general, la relación entre salarios y consumo energético se plantea de forma bidireccional; por un lado, los salarios definen la cantidad y calidad de calorías y nutrientes que consumen los hogares; y por otro, la cantidad y calidad de la alimentación puede influir sobre la productividad y los salarios. La mayoría de los estudios sobre nutrición y economía no abordan los problemas de endogeneidad presentes en la omisión de factores que afectan 
ambas variables; más bien se concentran en correlaciones entre variables como ingreso y consumo de calorías. La literatura sugiere que la ingesta de calorías y nutrientes está relacionada con la capacidad financiera de las familias (Drewnowski y Darmon, 2005; Hernández et al., 2017); sin embargo, se registra menos claridad sobre cómo es dicha ingesta a lo largo de la distribución de gasto en particular.

Respecto a la relación entre ingreso, consumo de calorías y padecimiento de enfermedades crónico-degenerativas, Drewnowski y Darmon (2005) afirman que las altas tasas de obesidad en Estados Unidos no son el resultado de una falla biológica, sino de un fenómeno sociodemográfico y económico. Ello se debe a que las personas de bajos ingresos suelen establecer el consumo de alimentos en términos de costo y sabor, dejando de lado otros aspectos como la variedad y el impacto de éstos sobre la salud. Cabe destacar que dichos resultados se concentran en el nivel socioeconómico en términos del ingreso, dejando de lado otras variables que pueden incidir en la ingesta energética.

En el caso de México, Hernández et al. (2017) encuentran que el costo de las calorías viene disminuyendo, que los patrones de consumo de alimentos varían a lo largo del tiempo, que los hogares más pobres prefieren alimentos con baja variabilidad en el precio y que obtienen la mayor parte de las calorías que consumen de alimentos con alta densidad calórica por lo que son más propensos a padecer malnutrición. Por otro lado, Martínez y Villezca (2005) muestran que los hogares más pobres no consiguen cubrir el total de sus requerimientos energéticos y nutricionales, a pesar de que usan más de la mitad de su ingreso corriente, mientras hogares más ricos satisfacen sus requerimientos proteínicos e incluso superan los calóricos con apenas una quinta parte de su ingreso.

Pérez y Minor (2011) muestran que, a la luz del encarecimiento de los alimentos durante la crisis de 2006 y 2008, los hogares rurales en México sustituyeron los alimentos costosos por otros más baratos, con mayor contenido energético y menor aportación nutrimental. El estudio analiza la composición del gasto en alimentos de un sector poblacional de referencia, para identificar cuáles son los alimentos que se consideran como básicos y analizar los cambios derivados de la crisis internacional de los alimentos. Destaca de este trabajo que incluye en su análisis información sobre algunos minerales y vitaminas a diferencia de estudios previos.

En México son escasos los estudios que incorporan la educación como elemento diferenciador del consumo de calorías y nutrientes. Experiencias en otros países sugieren que la escolaridad de los individuos puede significar un freno a la obesidad. Monteiro et al. (2001) para Brasil señala que una mayor 
escolaridad puede disminuir el riesgo de padecer obesidad, ya que los individuos pueden tomar mejores decisiones sobre su dieta. Por su parte, Harding et al. (2018) analizan la relación entre la escolaridad materna y el estatus del consumo de micronutrientes de sus hijos en varios países; los autores encuentran que la escolaridad materna está asociada a menores deficiencias en el estatus nutricional de los niños; sin embargo, señalan que los resultados varían según el nivel de ingreso de los países.

Esta revisión da cuenta de cómo la capacidad financiera está relacionada con el consumo energético y de micronutrientes, pero también de la existencia de heterogeneidad no observable en la relación entre estado nutricional y la posición socioeconómica familiar. Para el presente trabajo se parte de la idea de que la riqueza financiera por sí sola no representa de manera adecuada el estatus socioeconómico del hogar; por ello, se incluye la escolaridad del jefe de familia como información no sólo sobre el nivel socioeconómico, sino también sobre las ventajas, desventajas y capacidades de las familias. ${ }^{1}$

\section{IDENTIFICACIÓN: NUTRICIÓN EN LA ENIGH Y MODELOS ESTADÍSTICOS}

Este artículo estudia las cantidades de calorías y micronutrientes que se consumen en los hogares mexicanos según sus niveles gasto y la escolaridad del jefe de familia, con base en datos de la ENIGH-2018. Las calorías representan la cantidad de energía que el consumo de alimentos aporta para mantener el funcionamiento del organismo y llevar a cabo diversas tareas; mientras que los micronutrientes son elementos que forman parte de la dieta y en cantidades pequeñas resultan esenciales para un correcto funcionamiento del organismo. Existe evidencia de que un consumo excesivo o deficitario de energía y de micronutrientes puede tener consecuencias negativas asociadas con un menor desarrollo físico, cognitivo o incluso con el desarrollo de enfermedades crónico-degenerativas.

1 Se empleó la variable escolaridad del jefe de hogar bajo el supuesto de que ésta vincula al resto de integrantes en su acumulación de capital humano y de que la cabeza de familia tiene un papel activo en las decisiones de consumo del hogar. Frente a opciones como el promedio de escolaridad en el hogar, esta variable tiene la ventaja del ciclo de formación escolar concluido y generar menor dispersión de los datos. 
Owen Eli Ceballos Mina y Humberto Guadarrama Gómez

Tabla 1. Requerimiento energético por grupos de edad

\begin{tabular}{llll}
\hline Edad & Clasificación & Mujer & Hombre \\
\hline 1 a 11 años & Menores & 1510 & 1619 \\
12 a 17 años & Adolescentes & 2250 & 2810 \\
18 a 64 años & Adultos & 2219 & 2681 \\
65 años o más & Adultos mayores & 1950 & 2213 \\
\hline
\end{tabular}

Notas: kilocalorías relativas al promedio entre actividad física sedentaria y moderada.

Fuente: elaboración propia con base en Bonvecchio et al. (2015).

Tabla 2. Requerimiento de micronutrientes para adultos y adultos mayores

\begin{tabular}{|c|c|c|c|c|}
\hline \multirow[t]{2}{*}{ Micronutriente } & \multicolumn{2}{|c|}{ Adulto } & \multicolumn{2}{|c|}{ Adulto mayor } \\
\hline & Mujer & Hombre & Mujer & Hombre \\
\hline Vitamina A & $600 \mu g$ & $600 \mu g$ & $800 \mu g$ & $1,000 \mu g$ \\
\hline Vitamina C & $100-200 \mathrm{mg}$ & $100-200 \mathrm{mg}$ & $100-200 \mathrm{mg}$ & $100-200 \mathrm{mg}$ \\
\hline Hierro & $21 \mathrm{mg}$ & $9.1 \mathrm{mg}$ & $21 \mathrm{mg}$ & $9.1 \mathrm{mg}$ \\
\hline Calcio & $1,000-1,200 \mathrm{mg}$ & $1,000-1,200 \mathrm{mg}$ & $1,000-1,200 \mathrm{mg}$ & $1,000-1,200 \mathrm{mg}$ \\
\hline Zinc & $10 \mathrm{mg}$ & $10 \mathrm{mg}$ & $10 \mathrm{mg}$ & $10 \mathrm{mg}$ \\
\hline
\end{tabular}

Fuente: elaboración propia con base en Martínez-Puga y Lendoiro (2005).

Las tablas 1 y 2 muestran los consumos de calorías y micronutrientes, recomendados por especialistas, según el grupo etario y el sexo de la población. Los valores corresponden al promedio de las ingestas relativas a actividad física sedentaria y moderada, considerando la poca actividad física que muestra la población mexicana. Estas cantidades de referencia son importantes porque en los casos en los que no se satisfacen las ingestas sugeridas de micronutrientes, las personas pueden padecer malnutrición, independientemente de que las cuotas calóricas sean satisfechas. ${ }^{2}$

La estrategia de análisis de este ejercicio se centra en regresiones polinómicas no paramétricas y semiparamétricas que se controlan por las características

2 Los micronutrientes incluidos en este trabajo de forma conjunta influyen en la capacidad de memoria, atención y estimulación motora, en especial durante la infancia; véase Martínez-Puga y Lendoiro (2005), Rosado et al. (1999), FAO (2003). 
socioeconómicas y demográficas del hogar, tanto para el consumo de calorías como para el consumo de los cinco micronutrientes seleccionados. Se estiman modelos no paramétricos del consumo de calorías sobre las distribuciones de gasto y de los años de escolaridad del jefe de hogar como variables indicadoras de la riqueza y del capital humano de las familias. Posteriormente, se realizan regresiones semiparamétricas en dos etapas: en la primera, se estima el consumo de calorías y micronutrientes controlando por características demográficas y económicas; en la segunda, se realizan estimaciones no paramétricas como las del primer modelo, después de haber controlado por variables de la primera etapa.

\section{Nutrición de los hogares mexicanos en la ENIGH-2018}

Uno de los principales objetivos de la ENIGH es ser fuente de información detallada sobre el consumo de los hogares mexicanos. Para calcular el consumo de calorías y micronutrientes en este trabajo, se tomaron las cantidades de alimento del módulo de gasto de la encuesta y se multiplican por el aporte calórico y nutricional de cada alimento propuesto en las tablas de composición nutrimental de Muñoz de Chávez et al. (2010). ${ }^{3}$ El consumo entre hogares y al interior depende del número y la edad de sus miembros, por lo que se incorpora la escala de equivalencia paramétrica OCDE-Oxford ${ }^{4}$ propuesta por Mancero (2001) para obtener el consumo aparente diario equivalente de calorías y micronutrientes de cada hogar.

El módulo de gastos en alimentos y bebidas de la ENIGH-2018 incluye 39 categorías que se dividen en 236 subgrupos. La encuesta organiza dicha información en ocho grupos alimenticios: i) pan, tortillas y cereales; ii) carnes; iii) pescados y mariscos; iv) leche, derivados de leche y huevo; $v$ ) aceites $\mathrm{y}$ grasas vegetales; vi) frutas y hortalizas; vii) azúcar, café y refrescos envasados; y viii) otros alimentos. En este trabajo, en lugar de clasificar la población por

3 Esta metodología es desarrollada con base en Hernández et al. (2017). Las cantidades de alimento semanales se dividen entre siete y se multiplican por la cantidad de calorías o nutrientes de las tablas de nutrición disponibles en Muñoz de Chávez et al. (2010).

4 Esta escala determina la porción del consumo total que realiza cada miembro del hogar; asigna un valor de 1 al primer adulto, 0.7 por cada adulto adicional y 0.5 del primer adulto por cada nińo menor de 12 años.

5 Consumo aparente, ya que suele asumirse que las cantidades reportadas en la encuesta se consumen en su totalidad por los miembros del hogar, aunque no de manera homogénea (Martínez y Villezca, 2005). 
ingreso, se dividió en tres grupos por nivel de educación; i) baja escolaridad: hogares con jefes de familia con educación básica primaria o menos; ii) media escolaridad: hogares con cabezas de familia que hayan cursado más que primaria hasta preparatoria o menos; y iii) alta escolaridad: familias con jefes de hogar con estudios universitarios o de posgrado.

La tabla 3 muestra el monto del gasto en alimentos, su participación en el consumo total y las características sociodemográficas de los hogares mexicanos, por grupos de escolaridad. Se observa que $42 \%$ de los hogares son encabezados por jefes de familia con baja escolaridad; $45 \%$ de ellos cuentan con nivel secundaria o preparatoria y sólo 13\% alcanzan estudios superiores. La mayor parte de los hogares con baja escolaridad se encuentran en localidades rurales; la jefatura de hogar es mayoritariamente masculina sin importar la escolaridad, aunque en los hogares con baja escolaridad se registra mayor participación de mujeres cabezas de familia. Asimismo, el promedio de edad de los jefes es mayor en familias de baja escolaridad.

En cuanto al gasto mensual en alimentos, se identifica una clara correlación entre los hogares encabezados por personas con alta escolaridad y un mayor gasto mensual en alimentos pese a que la participación de dicho gasto, como proporción del total, cada vez es menor. El gasto en alimentos en los hogares de escolaridad alta es cerca del doble de los hogares con jefes con educación primaria o menos. Una primera evidencia acerca de las diferencias estructurales en el consumo por nivel de escolaridad es aquella verificación empírica atribuida a Engel en sus estudios sobre consumo (Engel y Kneip, 1996; García, 2013), en la que el gasto en alimentos es una proporción menor del gasto total a medida que se incrementa el ingreso.

La tabla 4 presenta la proporción de gasto y aporte calórico de los alimentos por nivel de escolaridad del jefe de familia agrupado por su densidad energética; los alimentos se clasificaron en grupos de alto, medio y bajo contenido calórico. ${ }^{6}$ La tabla muestra que la proporción de consumo de alimentos de alto contenido calórico y el monto de calorías consumidas disminuyen significativamente con el incremento de la escolaridad del jefe de hogar; también se observa un comportamiento inverso en términos del conjunto de alimentos de bajo contenido calórico que incrementa su proporción de consumo hasta en 20 puntos porcentuales entre los hogares con jefes de baja escolaridad y los hogares encabezados por personas con estudios superiores.

6 Con base en Hernández et al. (2017) se agruparon los alimentos por la cantidad de calorías que contienen en porción de 100 gramos o mililitros así: baja densidad energética de 4 a 145 calorías por cada $100 \mathrm{~g}$ o ml; media densidad energética de 145 a 300 calorías por cada $100 \mathrm{~g}$ o ml; y alta densidad energética de 300 a 900 calorías por cada $100 \mathrm{~g}$ o ml. 
Tabla 3. Gasto en alimentos y demografía por nivel de escolaridad del jefe

\begin{tabular}{lcccc}
\hline Características del hogar & $\begin{array}{c}\text { Baja } \\
\text { escolaridad }\end{array}$ & $\begin{array}{c}\text { Media } \\
\text { escolaridad }\end{array}$ & $\begin{array}{c}\text { Alta } \\
\text { escolaridad }\end{array}$ & Promedio \\
\hline Gasto mensual promedio en alimentos & $\$ 2871$ & $\$ 3769$ & $\$ 5652$ & $\$ 3750$ \\
$\begin{array}{l}\text { Gasto en alimentos como proporción del } \\
\text { gasto total }\end{array}$ & $46 \%$ & $41 \%$ & $33 \%$ & $42 \%$ \\
Jefatura masculina & $67 \%$ & $74 \%$ & $74 \%$ & $71 \%$ \\
Edad del jefe de hogar & 58.1 & 44.1 & 46.0 & 49.8 \\
Localidad rural & $37 \%$ & $18 \%$ & $5 \%$ & $23 \%$ \\
$\begin{array}{l}\text { Proporción de hogares por nivel } \\
\text { escolaridad del jefe }\end{array}$ & $42 \%$ & $45 \%$ & $13 \%$ & $100 \%$ \\
Observaciones & 31236 & 33312 & 10099 & 74647 \\
\hline
\end{tabular}

Fuente: cálculos propios con base en la ENIGH-2018.

Tabla 4. Proporción de gasto y aporte calórico diario por densidad energética de los alimentos y nivel de escolaridad del jefe de familia

\begin{tabular}{|c|c|c|c|c|c|c|c|c|}
\hline \multirow{2}{*}{$\begin{array}{l}\text { Densidad } \\
\text { energética }\end{array}$} & \multicolumn{2}{|c|}{ Baja escolaridad } & \multicolumn{2}{|c|}{ Media escolaridad } & \multicolumn{2}{|c|}{ Alta escolaridad } & \multicolumn{2}{|c|}{ Promedio } \\
\hline & $\begin{array}{c}\text { Proporción } \\
\text { del gasto }\end{array}$ & $\begin{array}{l}\text { Aporte } \\
\text { calórico }\end{array}$ & $\begin{array}{c}\text { Proporción } \\
\text { del gasto }\end{array}$ & $\begin{array}{l}\text { Aporte } \\
\text { calórico }\end{array}$ & $\begin{array}{l}\text { Proporción } \\
\text { del gasto }\end{array}$ & $\begin{array}{l}\text { Aporte } \\
\text { calórico }\end{array}$ & $\begin{array}{c}\text { Proporción } \\
\text { del gasto }\end{array}$ & $\begin{array}{l}\text { Aporte } \\
\text { calórico }\end{array}$ \\
\hline Alta & $21 \%$ & 1462 & $18 \%$ & 1055 & $13 \%$ & 940 & $18 \%$ & 1190 \\
\hline Media & $34 \%$ & 1175 & $31 \%$ & 1239 & $22 \%$ & 1107 & $30 \%$ & 1192 \\
\hline Baja & $45 \%$ & 417 & $51 \%$ & 458 & $65 \%$ & 578 & $52 \%$ & 463 \\
\hline Total & $100 \%$ & 3054 & $100 \%$ & 2752 & $100 \%$ & 2625 & $100 \%$ & 2845 \\
\hline
\end{tabular}

Fuente: cálculos propios con base en la ENIGH-2018.

Destaca que la mayor parte de las calorías consumidas por las familias con jefes de baja escolaridad provienen de alimentos con alta densidad energética, lo que por lo regular se asocia con el incremento de la probabilidad de consumo de alimentos con bajo impacto nutricional. Las familias con media y alta escolaridad satisfacen sus requerimientos calóricos con base en alimentos de media densidad energética. Cabe mencionar que estos hallazgos coinciden con los resultados de Hernández et al. (2017) para los años de 1992, 2000 y 
2010, lo que permite anticipar que no hay cambios importantes en los patrones de satisfacción de los requerimientos calóricos del país en cerca de los 30 años que cubren estos estudios.

En tanto, la tabla 5 presenta la proporción del gasto diario equivalente por grupo alimenticio y su respectivo aporte calórico. Se observan similitudes en la distribución del gasto de modo que los hogares asignan la mayor parte al consumo de carnes, harinas (pan, tortillas, cereales), vegetales y lácteos; sin embargo, también se observa una disminución del consumo de harinas conforme incrementa el nivel educativo del jefe de hogar acorde con la disminución de los alimentos de alta densidad energética. En cuanto al aporte calórico se observa que, a mayor nivel educativo menor es el consumo de calorías provenientes de harinas y azúcar; lo que sugiere que en hogares con mayor capital humano se privilegia el consumo de alimentos más ricos en nutrientes como pescados, frutas y hortalizas, frente al aporte calórico de estos alimentos; mientras que hogares con jefes de baja escolaridad sacrifican nutrientes en favor de alimentos con alto contenido calórico dado su precio.

La misma tabla sugiere que los diferentes estratos educativos se vinculan con la variedad en la dieta que consumen los individuos diariamente. Si bien la proporción del consumo de carne parece constante a lo largo del nivel de escolaridad del jefe de hogar, para el estrato de baja escolaridad, la mayor parte del consumo de calorías proviene de tres fuentes adicionales: harinas, azúcar, café y refrescos, y frutas y hortalizas. ${ }^{7}$ Para el estrato de media escolaridad, las fuentes de energía son las mismas que en el caso anterior, pero dan un peso adicional al consumo de lácteos y otros alimentos. Por último, el estrato de alta escolaridad revela que la cantidad de calorías se distribuye de manera más uniforme entre los grupos alimenticios.

7 La escasa variación en el consumo de carne, a lo largo de los niveles de escolaridad, puede explicarse en la relevancia de este grupo de alimentos en la dieta de los hogares mexicanos; sin embargo, proporciones de gasto similares (o incluso de ingesta calórica) no implican que los hogares consuman productos idénticos, sino que al interior de este grupo alimenticio pueden existir variaciones importantes del tipo de productos cárnicos y de la calidad de los mismos. 
Tabla 5. Proporción de gasto y aporte calórico diario por grupo de alimentos y nivel de escolaridad del jefe de familia

\begin{tabular}{|c|c|c|c|c|c|c|c|c|}
\hline \multirow{2}{*}{$\begin{array}{l}\text { Grupo de } \\
\text { alimentos }\end{array}$} & \multicolumn{2}{|c|}{ Baja escolaridad } & \multicolumn{2}{|c|}{ Media escolaridad } & \multicolumn{2}{|c|}{ Alta escolaridad } & \multicolumn{2}{|c|}{ Promedio } \\
\hline & $\begin{array}{c}\text { Proporción } \\
\text { del gasto }\end{array}$ & $\begin{array}{l}\text { Aporte } \\
\text { calórico }\end{array}$ & $\begin{array}{l}\text { Proporción } \\
\text { del gasto }\end{array}$ & $\begin{array}{l}\text { Aporte } \\
\text { calórico }\end{array}$ & $\begin{array}{c}\text { Proporción } \\
\text { del gasto }\end{array}$ & $\begin{array}{l}\text { Aporte } \\
\text { calórico }\end{array}$ & $\begin{array}{c}\text { Proporción } \\
\text { del gasto }\end{array}$ & $\begin{array}{l}\text { Aporte } \\
\text { calórico }\end{array}$ \\
\hline $\begin{array}{l}\text { Pan, tortilla } \\
\text { y cereales }\end{array}$ & $21 \%$ & 1186 & $18 \%$ & 831 & $13 \%$ & 638 & $18 \%$ & 933 \\
\hline Carnes & $22 \%$ & 227 & $24 \%$ & 266 & $23 \%$ & 266 & $23 \%$ & 251 \\
\hline $\begin{array}{l}\text { Pescados } \\
\text { y mariscos }\end{array}$ & $2 \%$ & 18 & $2 \%$ & 17 & $4 \%$ & 33 & $2 \%$ & 20 \\
\hline $\begin{array}{l}\text { Leche y } \\
\text { derivados }\end{array}$ & $13 \%$ & 264 & $14 \%$ & 307 & $14 \%$ & 354 & $14 \%$ & 299 \\
\hline $\begin{array}{l}\text { Aceites } \\
\text { y grasas }\end{array}$ & $2 \%$ & 216 & $1 \%$ & 163 & $1 \%$ & 116 & $1 \%$ & 175 \\
\hline $\begin{array}{l}\text { Frutas } \\
\text { y hortalizas }\end{array}$ & $19 \%$ & 345 & $17 \%$ & 286 & $18 \%$ & 309 & $18 \%$ & 312 \\
\hline $\begin{array}{l}\text { Azúcar, café } \\
\text { y refrescos }\end{array}$ & $12 \%$ & 586 & $12 \%$ & 624 & $11 \%$ & 573 & $12 \%$ & 601 \\
\hline $\begin{array}{l}\text { Otros } \\
\text { alimentos }\end{array}$ & $9 \%$ & 212 & $12 \%$ & 258 & $16 \%$ & 336 & $12 \%$ & 254 \\
\hline Total & $100 \%$ & 3054 & $100 \%$ & 2752 & $100 \%$ & 2625 & $100 \%$ & 2845 \\
\hline
\end{tabular}

Fuente: cálculos propios con base en la ENIGH-2018.

Las tablas presentadas en esta sección dan indicios sobre las relaciones entre el monto, la proporción de gasto y el contenido calórico por nivel de escolaridad; en general se evidencia que la relación entre escolaridad del jefe de hogar y el consumo energético equivalente es inversa, ya que las personas que pertenecen a familias con bajo nivel educativo consumen en promedio 3 054 calorías a diario, mientras que en los casos de media y alta escolaridad sus consumos son de 2752 y 2625 calorías, respectivamente; también se observa que dicha disminución del consumo de calorías se da conforme incrementa el nivel educativo del jefe y es acompañada de un incremento del monto de gasto en alimentos y de una menor participación de dicho gasto en el total de consumo (véase tabla 3); mientras los hogares con alta escolaridad gastan cerca de MXN\$188 diarios en 2625 calorías, los hogares con jefes menos educados gastan sólo MXN\$96 para consumir 3054 calorías diarias en promedio. 
Los hogares con menor capital humano acumulado dedican mayor proporción del gasto y consumen más calorías que provienen de alimentos con alto contenido energético y bajo impacto nutricional; estos productos menos convenientes en el proceso nutricional hacen a los miembros de estos hogares más propensos a padecer sobrepeso u obesidad como sugieren Hernández et al. (2017). En contraste, los hogares de media y alta escolaridad prefieren reducir la ingesta calórica derivada de harinas y azúcares para darle más peso a otros alimentos como pescados, lácteos y vegetales que resultan más ricos en micronutrientes, disminuyendo así la propensión al desarrollo de enfermedades asociadas con la malnutrición. En estos descriptivos se tienen indicios claros de que las desigualdades educativas en el país participan activamente en la explicación de las desigualdades nutricionales.

\section{Modelos no paramétricos y semiparamétricos}

Para estimar los efectos de la escolaridad sobre el consumo energético y de nutrientes de los hogares se observa el comportamiento del consumo de calorías, hierro, calcio, zinc y vitaminas A y C sobre el nivel de educación del jefe de hogar y la distribución de gasto, sin asignar una forma funcional preconcebida. Se estiman modelos no paramétricos como los usados por Delgado y Miles (1997) y García (2012), usando estimadores polinómicos derivados de una función Kernel:

$$
\text { nutr }_{i}=m\left(\text { edujh }_{i} / \text { gast }_{i}\right)+\varepsilon_{i}
$$

La variable nutr $_{i}$ en la ecuación (1) refiere al consumo de calorías, hierro, calcio, zinc, vitaminas A o C del hogar $i$. El residual $\varepsilon_{i}$ es aleatorio e independiente de la variable explicativa. La función $m\left(e d u j h_{i} / g_{\text {ast }}\right)$ es una función no paramétrica de los ańos de educación del jefe de hogar $\left(e d u j h_{i}\right)$ o del logaritmo del gasto ( gast $_{i}$ ) del hogar $i$, y se estima usando polinomios locales. Si se define la variable $X_{j}=e d u j h_{j}$ o $X_{j}=g_{a s t}$, entonces para un nivel de educación o nivel de gasto $x_{o}$ de la ecuación nutr $_{j}=m\left(X_{j}\right)+\epsilon_{j}$ se tiene una aproximación $m\left(x_{0}\right)$ :

$$
\operatorname{argmin} m\left(x_{o}\right)=\sum_{j=1}^{N} F_{j}^{2} K\left(X_{j}-x_{o}\right)
$$

donde $K$ es una función Kernel que da mayor peso a los hogares cuya edad del jefe (o gasto familiar) tiene valores cercanos a $x_{o}$. Con base en la propuesta 
de Gutierrez et al. (2003), la ecuación (2) para cada punto $x_{o}$ de los años de educación o del logaritmo del gasto, estima una aproximación $m\left(x_{o}\right)$, por lo que la función $m\left(e d u j h_{i} /\right.$ gast $\left._{i}\right)$ resulta muy flexible para capturar variaciones del consumo de calorías y micronutrientes sobre la escolaridad y el nivel económico de los hogares. ${ }^{8}$

Como una estrategia de contraste para dilucidar los efectos del nivel de escolaridad del jefe de hogar sobre el comportamiento del consumo de calorías y de micronutrientes a lo largo del nivel socioeconómico del hogar, se estiman regresiones semiparamétricas usando un modelo lineal parcial en dos etapas como el propuesto por Speckman (1988); el método combina estimadores paramétricos que se obtienen a partir de mínimos cuadrados ordinarios (MCO) y estimadores no paramétricos derivados de una función Kernel como los expuestos en la ecuación (1) de la primera parte de este ejercicio de identificación. La regresión semiparamétrica general por estimar es:

$$
n u t r_{i}=\pi X_{i}+m\left(e^{e d u j h_{i}} / \text { gast }_{i}\right)+\varepsilon_{i t}
$$

Igual que en la ecuación (1), la variable nutr $_{i}$ refiere al consumo de calorías, hierro, calcio, zinc, vitaminas A o C del hogar $i$; y $m\left(e d u j h_{i} /\right.$ gast $\left._{i}\right)$ es función no paramétrica de los años de educación del jefe de familia o del logaritmo del gasto del hogar, que se estima usando polinomios locales como se explicó en la ecuación (2) del modelo no paramétrico. $X_{i}$ es un vector de características que incluye el sexo, la edad y la edad al cuadrado del jefe de familia; el número de integrantes y ocupados del hogar; si el hogar se encuentra en una localidad urbana o rural; la participación de los alimentos en el total de gastos familiares; e incluye un control sobre el decil de gasto (regresión sobre la escolaridad) o los años de escolaridad del jefe de hogar (regresión sobre el logaritmo del gasto). Finalmente, el residual $\varepsilon_{i}$ es aleatorio e independiente de las variables explicativas.

Una diferencia importante entre la regresión no paramétrica de la ecuación (1) y la regresión semiparamétrica en la ecuación (3) es que la última se estima en dos etapas; en la primera se calculan de forma paramétrica los efectos de la escolaridad del jefe de hogar sobre el consumo de calorías y micronutrientes controlando por todas las características del vector $X_{i}$; en la segunda se estiman de forma no paramétrica los efectos no lineales de la escolaridad y del

8 Gutierrez et al. (2003) plantean: $\operatorname{argmin} m\left(x_{o}\right)=\sum_{t=1}^{N}\left\{\mathrm{Yi}-\sum_{i=0}^{p} \beta_{j}\left(X_{i}-x_{o}\right)^{j}\right\}^{2} K\left(X_{i}-x_{o}\right)$. La ecuación (2) fija el grado de polinomio $p$ en 0 y usa una función Kernel Epanechnikov; una configuración común en la aplicación de este tipo de modelos. 
nivel económico sobre el consumo de calorías y de micronutrientes, después de controlar por las características del hogar, es decir, usando los valores predichos en la primera etapa.

Las regresiones propuestas en las ecuaciones (1) y (3) ofrecen la ventaja de su eficiencia para capturar los efectos no lineales del nivel de educación y del nivel económico sobre el consumo de calorías y micronutrientes, sin imponer restricciones y suposiciones previas sobre el comportamiento de los datos. La pérdida de eficiencia de la estimación en dos etapas del modelo semiparamétrico frente a una etapa única es justificada dadas las ventajas de la metodología lineal parcial para capturar los efectos no lineales del nivel socioeconómico sobre el consumo calórico y de micronutrientes.

\section{PERFILES DE CONSUMO ENERGÉTICO Y DE MICRONUTRIENTES}

En esta sección se presentan los resultados gráficos de las estimaciones no paramétricas y semiparamétricas propuestas en la estrategia de identificación. La sección se divide en dos subapartados; en el primero se presentan los perfiles del consumo de calorías sobre los años de educación del jefe de familia y sobre el logaritmo de gasto del hogar; en el segundo se hace la revisión de los perfiles del consumo de minerales y vitaminas sobre el nivel socioeconómico del hogar representado en la escolaridad del jefe y el nivel de gasto.

\section{Escolaridad del jefe de familia y nivel de gasto del hogar sobre el consumo equivalente de calorías diarias en el hogar}

La gráfica 1 en sus dos paneles muestra las estimaciones no paramétricas de los perfiles del consumo aparente de kilocalorías diarias ( $\mathrm{kcd}$ ) sobre el nivel socioeconómico de las familias mexicanas; del lado izquierdo se observa el consumo equivalente de $\mathrm{kcd}$ a lo largo del logaritmo del gasto como una representación del nivel económico de las familias; del lado derecho se observa cómo cambia dicho consumo de kcd con los años de educación del jefe de familia como representación del capital humano en los hogares; en ambos casos se modela el consumo energético sin imponer ninguna restricción adicional sobre su comportamiento tal y como se propuso en la ecuación (1). 
Gráfica 1. Consumo energético por nivel de gasto y educación

Modelos no paramétricos
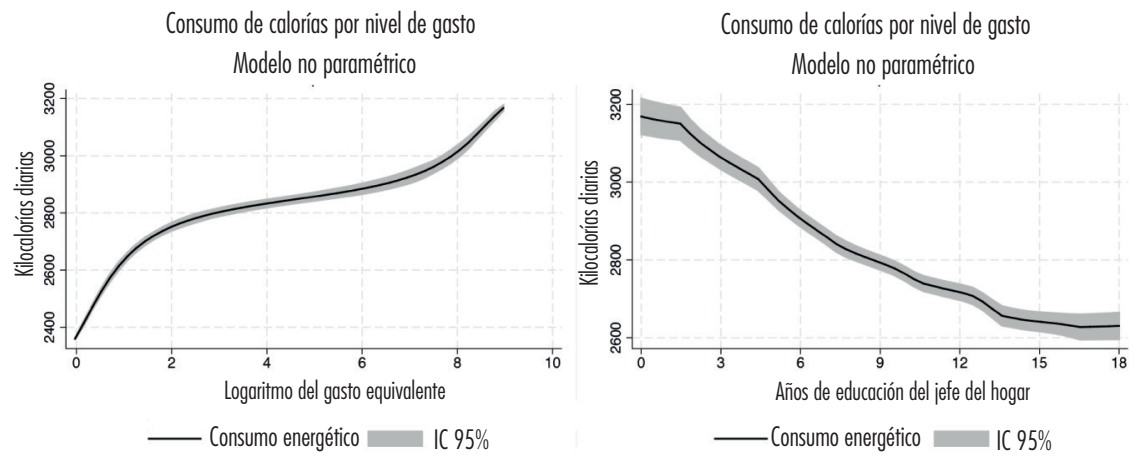

Fuente:cálculos propios con datos de la ENIGH-2018.

Las estimaciones no paramétricas muestran que el consumo de calorías aumenta desde 2400 hasta $3200 \mathrm{kcd}$ con el incremento del nivel económico a lo largo del logaritmo del gasto de los hogares; se identifica que familias con bajo nivel económico no alcanzan los umbrales de consumo energético recomendados, mientras dichos umbrales son superados en el extremo opuesto denotando efectos de la doble carga de malnutrición. Del lado derecho, con el incremento de los años de educación del jefe de familia, el consumo diario equivalente de calorías disminuye desde $3200 \mathrm{kcd}$ por persona en hogares con cabezas de familia con bajo nivel educación, hasta alrededor de $2600 \mathrm{kcd}$ conforme el nivel de escolaridad del jefe de hogar se acerca a estudios superiores.

Estos comportamientos opuestos del consumo de calorías a lo largo del nivel educativo y del nivel gasto, representan los efectos directos y sin controles adicionales del nivel económico de los hogares y su acumulación de capital humano; de este modo, mientras el consumo de calorías por persona en el hogar aumenta con el incremento del nivel económico familiar, disminuye con los años de educación del jefe de hogar, de forma que incluso se aproxima a los umbrales recomendados por los organismos internacionales.

Como ya se mencionó, son muchas las variables que intervienen en la determinación del consumo energético del hogar; una cuestión inmediata es si los comportamientos del consumo de calorías, a lo largo del nivel de gasto y de educación del jefe de familia del modelo no paramétrico, se mantienen después de controlar por otros factores sociodemográficos como se propuso con la estrategia semiparamétrica. 
Tabla 6. Consumo de calorías sobre el nivel de gasto y de educación Modelos semiparamétricos-Primera etapa

\begin{tabular}{|c|c|c|}
\hline $\begin{array}{c}\text { Controles } \\
\text { sociodemográficos }\end{array}$ & $\begin{array}{l}\text { (1) } \\
\text { Consumo de energético sobre la } \\
\text { educación } \\
(k c d)\end{array}$ & $\begin{array}{c}\text { (2) } \\
\text { Consumo de energético } \\
\text { sobre el gasto } \\
(\mathrm{kcd})\end{array}$ \\
\hline \multirow[t]{2}{*}{ Localidad rural } & $506.15^{\star \star \star}$ & $436.35^{\star \star \star}$ \\
\hline & $(16.93)$ & $(17.47)$ \\
\hline \multirow[t]{2}{*}{ Número de integrantes del hogar } & $-168.12^{\star \star \star}$ & $-171.56^{\star \star \star}$ \\
\hline & (5.33) & (5.59) \\
\hline \multirow[t]{2}{*}{ Número de ocupados en el hogar } & $106.42^{\star \star \star}$ & $87.10^{\star \star \star}$ \\
\hline & $(8.00)$ & (8.48) \\
\hline \multirow[t]{2}{*}{ Jefatura masculina } & 12.14 & $98.55^{\star \star \star}$ \\
\hline & $(16.67)$ & $(17.53)$ \\
\hline \multirow[t]{2}{*}{ Edad del jefe de hogar } & $33.69^{\star \star \star}$ & $30.97^{\star \star \star}$ \\
\hline & (3.08) & $(3.21)$ \\
\hline \multirow[t]{2}{*}{ Edad al cuadrado } & $-0.25^{\star \star \star}$ & $-0.30^{\star \star \star *}$ \\
\hline & $(0.03)$ & $(0.03)$ \\
\hline \multirow[t]{2}{*}{ Proporción de alimentos en el total gasto } & $57.92^{\star \star \star}$ & $40.22^{\star \star \star}$ \\
\hline & (0.69) & $(0.61)$ \\
\hline \multirow[t]{2}{*}{ Logaritmo del ingreso per cápita del hogar } & $-319.38^{\star \star \star}$ & $345.66^{\star \star *}$ \\
\hline & (15.33) & $(15.16)$ \\
\hline \multirow[t]{2}{*}{ Logaritmo del gasto monetario del hogar } & $831.43^{\star \star \star}$ & 2da. Etapa \\
\hline & $(12.67)$ & \\
\hline \multirow[t]{2}{*}{ Años de educación del jefe de hogar } & 2da. ełapa & $-16.24^{\star \star \star}$ \\
\hline & & (2.12) \\
\hline \multirow[t]{2}{*}{ Constante } & $-1,652.02^{\star \star \star}$ & $-781.05^{\star \star \star}$ \\
\hline & & $(105.82)$ \\
\hline Observaciones & 74,573 & 74,573 \\
\hline R-cuadrado & 0.2083 & 0.1287 \\
\hline
\end{tabular}

Notas: ${ }^{\star \star \star} p<0.01 ;{ }^{* \star} p<0.05 ;{ }^{\star} p<0.1$ Errores estándar robustos en paréntesis.

Fuente: cálculos propios con datos de la ENIGH-2018. 
La tabla 6 presenta las estimaciones MCo de la primera etapa de los modelos del consumo energético (1) sobre la escolaridad del jefe de hogar y (2) sobre el nivel de gasto de los hogares. Se controló por la localización geográfica de los hogares, identificando que personas en zonas rurales en promedio consumen entre 436 y 506 kcd más que aquellos individuos en zonas urbanas. Un integrante adicional en el hogar disminuye el consumo energético por individuo alrededor de $170 \mathrm{kcd}$, mientras un ocupado adicional en la familia incrementa dicho consumo en alrededor de $100 \mathrm{kcd}$. Efectos positivos más pequeños se identifican con la jefatura masculina del hogar y con el incremento de la edad del cabeza de familia.

Al controlar el consumo de calorías por el ingreso per cápita del hogar en la primera etapa de los modelos semiparamétricos aparecen efectos diferenciados dada la alta correlación del ingreso con el gasto familiar; un incremento de $1 \%$ el ingreso disminuye en $3.19 \mathrm{kcd}$ el consumo equivalente en el modelo semiparamétrico sobre la educación; mientras que aumenta dicho consumo energético en $3.45 \mathrm{kcd}$ cuando el gasto no se incluye en la ecuación del modelo semiparamétrico del nivel económico. El gasto y la educación del jefe de familia confirman efectos positivos y negativos, respectivamente, tal como lo indicaban los modelos no paramétricos. En general, los efectos de la primera etapa en ambos casos muestran comportamientos bastante intuitivos y con los signos esperados alrededor de los efectos sociodemográficos sobre el consumo energético.

Después de controlar en la primera etapa por el conjunto de factores sociodemográficos (véase tabla 6), la gráfica 2 presenta los resultados gráficos del modelo semiparamétrico en la segunda etapa; del lado izquierdo muestra que

Gráfica 2. Consumo energético sobre el nivel de gasto y de educación
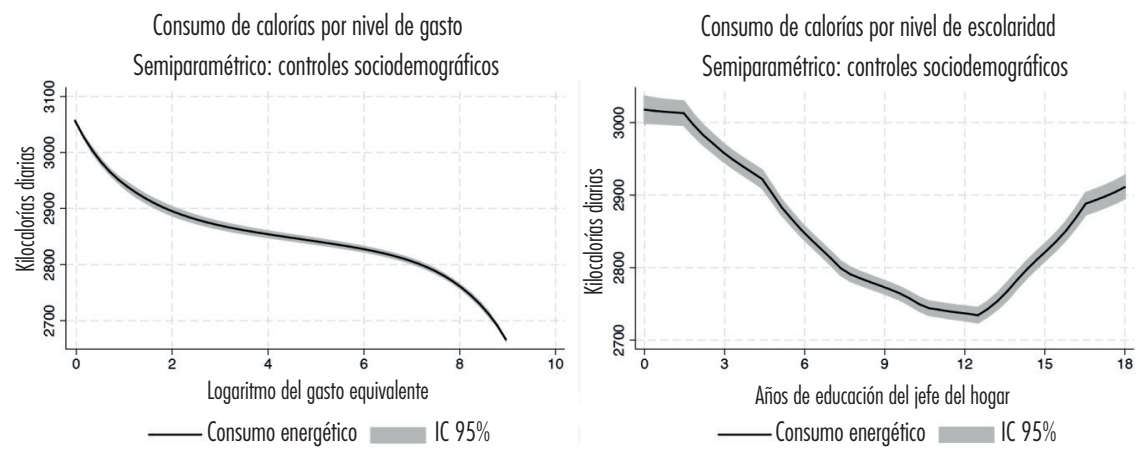

Fuente: cálculos propios con datos de la ENIGH-2018. 
el consumo de calorías de los hogares mexicanos a lo largo del logaritmo del gasto tiene un comportamiento decreciente desde 3100 hasta cerca de 2600 kcd; es decir, una vez incorporados controles sociodemográficos en el análisis del consumo energético sobre el nivel gasto, se observa que a mayor nivel económico el consumo de kcd por persona se acerca a los niveles recomendados para una buena nutrición. En el caso del consumo energético a lo largo del nivel de la escolaridad del jefe de hogar, después de incorporar los controles de la primera etapa, el modelo semiparamétrico muestra que el comportamiento del consumo equivalente de calorías mantiene una pendiente negativa en principio; es decir, a mayor educación menor consumo calórico, igual que en el caso no paramétrico; sin embargo, después de que el jefe completa estudios de nivel preparatorio el comportamiento cambia, generando una forma de "U" del consumo energético a lo largo del nivel educativo.

\section{Escolaridad del jefe de familia sobre el consumo de micronutrientes}

A continuación se presentan los perfiles de consumo de vitaminas y minerales de los hogares mexicanos a lo largo de la escolaridad el jefe de hogar de forma semiparamétrica, es decir, después de controlar en la primera etapa por variables sociodemográficas igual que se hizo con el consumo de calorías.

La tabla 7 presenta las estimaciones MCo del consumo en miligramos (mg) y microgramos $(\mu \mathrm{g})$ diarios de hierro, calcio, zinc, vitamina A y vitamina C, cada uno explicado por las características sociodemográficas de los hogares. Como ocurre con el consumo energético, se observa que el consumo equivalente de los cinco micronutrientes en localidades rurales es mayor que en localidades urbanas, mientras que con el incremento del tamaño del hogar dicho consumo disminuye. El incremento del número de ocupados en el hogar aumenta el consumo de micronutrientes con excepción del consumo de calcio y de vitamina $\mathrm{C}$ que disminuyen en 22.60 y $2.87 \mathrm{mg}$, respectivamente.

Destaca en esta primera etapa del modelo el consumo de micronutrientes sobre el gasto y la escolaridad del jefe de familia que, si bien la jefatura masculina incrementa el consumo energético del hogar, claramente disminuye el de los tres minerales y las dos vitaminas analizadas. El resto de los factores sociodemográficos se comporta con efectos en la misma dirección del consumo calórico; es decir, con el aumento de la edad del jefe de familia, de la proporción del gasto en alimentos y del gasto total del hogar se incrementa el consumo de micronutrientes, mientras que con el incremento del ingreso per cápita disminuyen dichos consumos a excepción de la ingesta de vitamina C. 
Tabla 7. Consumo de micronutrientes sobre la educación del jefe de familia Modelos semiparamétricos-Primera etapa

\begin{tabular}{|c|c|c|c|c|c|}
\hline $\begin{array}{l}\text { Controles } \\
\text { Sociodemográficos }\end{array}$ & $\begin{array}{c}\text { (1) } \\
\text { Consumo } \\
\text { de hierro } \\
\text { (mg) }\end{array}$ & $\begin{array}{c}\text { (2) } \\
\text { Consumo } \\
\text { de calcio } \\
\text { (mg) }\end{array}$ & $\begin{array}{c}\text { (3) } \\
\text { Consumo } \\
\text { de zinc } \\
(m g)\end{array}$ & $\begin{array}{c}(4) \\
\text { Consumo } \\
\text { vitamina A } \\
(\mu g)\end{array}$ & $\begin{array}{c}\text { (5) } \\
\text { Consumo } \\
\text { vitamina C } \\
(\mathrm{mg})\end{array}$ \\
\hline \multirow[t]{2}{*}{ Localidad rural } & $2.56^{\star \star \star}$ & $92.28^{\star \star \star}$ & $1.37^{\star \star \star}$ & $33.44^{\star \star \star}$ & $5.30^{\star \star \star}$ \\
\hline & $(0.21)$ & (6.84) & $(0.17)$ & (8.89) & (1.42) \\
\hline \multirow{2}{*}{$\begin{array}{l}\text { Número de integrantes } \\
\text { del hogar }\end{array}$} & $-2.11^{\star \star \star}$ & $-25.25^{\star \star \star}$ & 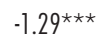 & $-35.92^{\star \star \star}$ & -0.56 \\
\hline & $(0.08)$ & (2.16) & $(0.06)$ & (2.63) & $(0.38)$ \\
\hline \multirow{2}{*}{$\begin{array}{l}\text { Número de ocupados } \\
\text { en el hogar }\end{array}$} & $1.55^{\star \star \star}$ & $-22.60^{\star \star \star}$ & $1.10^{\star \star \star}$ & $31.04^{\star \star \star}$ & $-2.87^{\star \star \star}$ \\
\hline & $(0.11)$ & (3.31) & $(0.09)$ & (4.19) & $(0.62)$ \\
\hline \multirow[t]{2}{*}{ Jefatura masculina } & $-0.97^{\star \star \star}$ & $-48.93^{\star \star \star}$ & -0.17 & $-51.80^{\star \star \star}$ & $-10.86^{\star \star *}$ \\
\hline & $(0.20)$ & (6.83) & $(0.16)$ & $(9.00)$ & (1.22) \\
\hline \multirow[t]{2}{*}{ Edad del jefe de hogar } & $0.33^{\star \star \star}$ & $11.72^{\star \star \star}$ & $-0.18^{\star \star \star}$ & $14.43^{\star \star \star}$ & $2.38^{\star \star \star}$ \\
\hline & $(0.03)$ & (1.08) & $(0.02)$ & (1.43) & $(0.21)$ \\
\hline \multirow[t]{2}{*}{ Edad al cuadrado } & $-0.00^{\star \star \star}$ & $-0.07^{\star \star \star}$ & $-0.00^{\star \star \star}$ & $-0.10^{\star \star \star}$ & $-0.02^{\star \star \star}$ \\
\hline & $(0.00)$ & $(0.01)$ & $(0.00)$ & $(0.01)$ & $(0.00)$ \\
\hline \multirow{2}{*}{$\begin{array}{l}\text { Proporción de alimentos } \\
\text { en el total gasto }\end{array}$} & $0.48^{\star \star \star}$ & $16.85^{\star \star \star}$ & $0.31^{\star \star \star}$ & $14.36^{\star \star \star}$ & $2.03^{\star \star \star}$ \\
\hline & $(0.01)$ & $(0.24)$ & $(0.01)$ & $(0.30)$ & $(0.05)$ \\
\hline \multirow{2}{*}{$\begin{array}{l}\text { Logaritmo del ingreso } \\
\text { per cápita del hogar }\end{array}$} & $-4.38^{\star \star \star}$ & $-85.95^{\star \star \star}$ & $-2.40^{\star \star \star}$ & $-70.18^{\star \star \star}$ & $7.81^{\star \star \star}$ \\
\hline & $(0.24)$ & (5.70) & $(0.21)$ & (7.18) & (1.09) \\
\hline \multirow{2}{*}{$\begin{array}{l}\text { Logaritmo del gasto } \\
\text { monetario del hogar }\end{array}$} & $7.11^{\star \star \star}$ & $256.90^{\star \star \star}$ & $4.82^{\star \star \star}$ & $220.67^{\star \star \star}$ & $37.47^{\star \star \star}$ \\
\hline & $(0.18)$ & (4.38) & $(0.15)$ & (5.93) & $(0.91)$ \\
\hline \multirow[t]{2}{*}{ Constante } & $-3.10^{\star \star}$ & $-477.29^{\star \star \star}$ & $-3.84^{\star \star \star}$ & $-682.08^{* \star *}$ & $-223.83^{\star \star}$ \\
\hline & & $(38.21)$ & $(4.24)$ & $(52.63)$ & (8.78) \\
\hline Observaciones & 74,573 & 74,573 & 74,573 & 74,573 & 74,573 \\
\hline R-cuadrado & 0.1065 & 0.1166 & 0.0750 & 0.0546 & 0.0728 \\
\hline
\end{tabular}

Notas: ${ }^{\star \star \star} p<0.01 ;{ }^{* \star} p<0.05 ;{ }^{\star} p<0.1$ Errores estándar robustos en paréntesis.

Fuente: cálculos propios con datos de la ENIGH-2018. 
Finalmente, la gráfica 3 presenta los perfiles de consumo equivalente de hierro, calcio, zinc, vitamina $\mathrm{A}$ y vitamina $\mathrm{C}$ a lo largo de la escolaridad del cabeza de familia. Similar al comportamiento del consumo de calorías sobre el nivel de educación, el consumo de minerales (hierro, calcio, zinc) y de vitamina A, tiene forma de " $U$ "; en principio, con el aumento de los años de escolaridad del jefe de hogar, el consumo de minerales y de vitamina A tienen un comportamiento decreciente hasta que la escolaridad sobrepasa el nivel de educación preparatoria (12 años) y el consumo de hierro, calcio y zinc empieza a elevarse con el aumento de la escolaridad.

En el caso particular del consumo equivalente de vitamina C, el perfil sencillamente es creciente con el incremento de los años de educación del jefe de familia, con una marcada aceleración del consumo de esta vitamina después de que el cabeza de familia supera los 12 años promedio de escolaridad y se inserta en estudios de nivel superior y posgrado.

Gráfica 3. Consumo de micronutrientes por nivel de educación

Modelos semiparamétricos - Segunda etapa
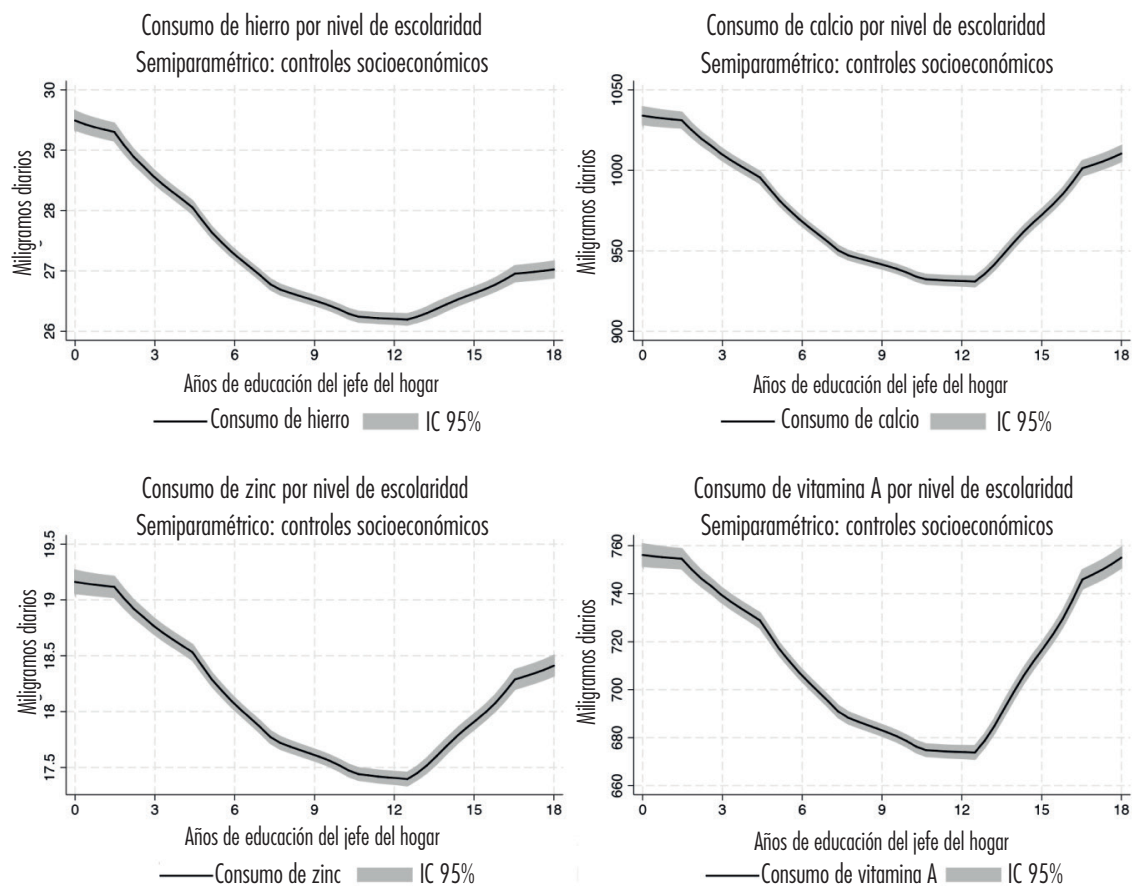
Gráfica 3. Consumo de micronutrientes por nivel de educación Modelos semiparamétricos - Segunda etapa (continuación)

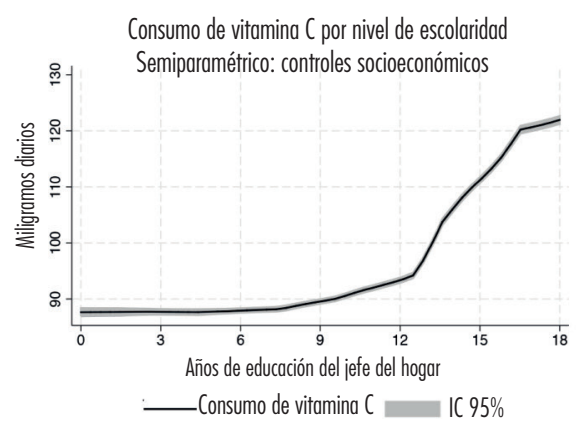

Fuente: cálculos propios con datos de la ENIGH-2018.

\section{CONCLUSIONES}

Este estudio aportó evidencia estadística acerca de los factores que determinan el consumo de energía y de micronutrientes en los hogares mexicanos. Con el fin de estudiar la relación entre nivel socioeconómico y nutrición, se estiman modelos semiparamétricos del consumo de calorías y de micronutrientes sobre un conjunto de variables representativas de la riqueza y el capital humano de las familias; para ir más allá de la dimensión monetaria y lo acostumbrado por la literatura, el análisis hace énfasis en la identificación de los efectos de la escolaridad del jefe de hogar sobre el comportamiento del consumo calórico y de micronutrientes. Los resultados indican que la escolaridad actúa como un modificador de la alimentación de los hogares a lo largo de la distribución de gastos familiares; en particular, con el incremento del logro educativo del jefe del hogar después de la formación preparatoria, las familias controlan el consumo excesivo de calorías, mientras incrementan el consumo de micronutrientes como hierro, calcio, zinc y vitaminas A y C.

El trabajo también aportó evidencia estadística de la importancia de la capacidad financiera para explicar la configuración del consumo alimentario y nutricional de las familias mexicanas. En general, el consumo de calorías y micronutrientes se incrementa con la riqueza monetaria de los hogares cuando no se introducen controles adicionales (modelo no paramétrico); sin embargo, una vez que se toman en cuenta las variables sociodemográficas del hogar (modelo semiparamétrico), las familias con bajos ingresos, ubicadas en la parte baja de la distribución del gasto monetario, consumen dietas que por exceso 
de calorías y por déficit de micronutrientes están alejadas de los niveles recomendados; mientras, los hogares con altos ingresos consumen dietas variadas y cercanas a los niveles que recomiendan los expertos y organismos de salud.

Los resultados evidencian que el nivel de riqueza familiar puede afectar el consumo de energía y de micronutrientes según el logro académico alcanzado por el cabeza de familia. Cuando no se imponen restricciones, el modelo no paramétrico muestra que el consumo de calorías siempre disminuye con el incremento de los años de educación del jefe de hogar; pero, incluso después de controlar por variables sociodemográficas, incluyendo la riqueza en el modelo semiparamétrico, se confirma que con el incremento del nivel de educación del jefe disminuye el consumo de calorías; esto ocurre al menos hasta llegar al nivel de educación preparatoria, porque después de los 12 años de educación formal se registra un leve repunte del consumo energético, generando una distribución en forma de "U". Asimismo, la forma de "U" sobre los años de educación es un comportamiento que se replica en el modelo semiparamétrico para el caso del consumo de micronutrientes con excepción del consumo de vitamina $\mathrm{C}$ que es creciente a lo largo de toda la distribución.

Del conjunto de resultados del análisis se destacan al menos tres implicaciones; por un lado, la limitada capacidad de la riqueza (representada en el gasto monetario) para determinar por sí sola el consumo energético y de micronutrientes de los hogares; segundo, el papel fundamental del capital humano, en particular del nivel educativo, como determinante de los resultados alimentarios y nutricionales de las familias mexicanas; y por último, la existencia de una acumulación de ventajas y desventajas basadas en la correlación entre la dimensión educativa y los estados nutricionales de las familias. Al respecto, se presentó evidencia de que el incremento del ingreso y los gastos del hogar, en ausencia del capital humano, no necesariamente conduce a niveles nutricionales adecuados.

Por último, es importante destacar que el nivel de educación alcanzado por el jefe de familia genera efectos diferenciados sobre el consumo energético y de micronutrientes del hogar. En particular, estudios preparatorios y superiores generan mejores resultados nutricionales, por lo que un fortalecimiento de la escolaridad repercute favorablemente en la salud de las personas, vía mejores hábitos alimenticios. En cambio, los niveles académicos asociados con menores niveles de escolaridad repercuten negativamente en la configuración de la dieta, generando resultados negativos que podrían desencadenar la misma dirección en otras dimensiones de vida. Bajo la reflexión previa y ante los alarmantes niveles de sobrepeso, obesidad y desnutrición que se observan en el país, resulta inaplazable revisar e incentivar el diseño de políticas públicas 
direccionadas sobre los niveles de escolaridad en hogares vulnerables, y a su vez el consumo de dietas equilibradas y variadas, a fin de romper y evitar la formación de desventajas en dichos hogares.

\section{BIBLIOGRAFÍA}

Bonvecchio, A., Fernández, A., Belausteguigoitia, M., Kaufer, M., Pérez, A. y Rivera, J. (2015), Guías alimentarias y de actividad física en contexto de sobrepeso y obesidad de la población mexicana, Ciudad de México, CONACYT.

Consejo Nacional de Evaluación de la Política de Desarrollo Social (coneval) (2015), "Diagnóstico sobre alimentación y nutrición: Informe Ejecutivo", Diagnóstico sobre alimentación y nutrición, Ciudad de México, Coneval. Recuperado de <https://www.coneval.org.mx/Evaluacion/ECNCH/Docu ments/Diagnostico_sobre_alimentacion_y_nutricion_270715.pdf>

Delgado, M. A. y Miles, D. (1997), "Household characteristics and consumption behaviour: A nonparametric approach", Empirical Economics, vol. 22, núm. 3, DOI <https://doi.org/10.1007/BF01208831>

Drewnowski, A. y Darmon, N. (2005), "Food choices and diet costs: An economic analysis", The Journal of Nutrition, núm. 135, DOI <https://doi. org/10.1017/jfm.2011.395>

y Specter, S. (2004), "Poverty and obesity: The role of energy density and energy costs", American Journal of Clinical Nutrition, vol. 79, núm. 1, DOI <https://doi.org/10.1093/ajcn/79.1.6>

Engel, J. y Kneip, A. (1996), "Recent approaches to estimating Engel curves", Journal of Economics, vol. 63, núm. 2, DOI <https://doi.org/10.1007/ BF01258672>

ENIGH-INEgI (2018), Encuesta Nacional de Ingresos y Gastos de los Hogares 2018, Instituto Nacional de Estadística y Geografía. Recuperado de $<$ https://www.inegi.org.mx/programas/enigh/nc/2018/>

faO (2003), "Perfiles nutricionales por países: México", Perfiles nutricionales por paises, Roma, FAO. Recuperado de <http://www.fao.org/3/aq028s/ aq028s.pdf>

Fernández, A., Martínez, R., Carrasco, I. y Palma, A. (2017), Impacto social y económico de la doble carga de la malnutrición, Santiago de Chile, CEPAL. Recuperado de <http://es.wfp.org/sites/default/files/es/file/espanol_estudiopiloto_abril_2017.pdf>

García, R. (2012), "Medidas de desempeño en regresiones no paramétricas de curvas de Engel", Comunicaciones En Estadística, vol. 5, núm. 2. 
Recuperadode $<$ https://www.academia.edu/4217271/Medidas_de_Desem pe\%C3\%B1o_en_Regresiones_No_Param\%C3\%A9tricas_de_Curvas_ de_Engel>

(2013), "Sobre las curvas de Engel: Una breve revisión de su evolución histórica”, Ensayos de Economía, vol. 22, núm. 42. Recuperado de $<$ https://revistas.unal.edu.co/index.php/ede/article/view/41258>

Gutierrez, R., Linhart, J. M. y Pitblado, J. (2003), "From the help desk : Local polynomial", The Stata Journal, vol. 3, núm. 4. Recuperado de <https:// ideas.repec.org/a/tsj/stataj/v3y2003i4p412-419.html>

Harding, K., Aguayo, V., Masters, W. y Webb, P. (2018), "Education and micronutrient deficiencies: An ecological study exploring interactions between women's schooling and children's micronutrient status", $B M C P u$ blic Health, vol. 18, núm. 1, DOI <https://doi.org/10.1186/s12889-0185312-1>

Hernández, G., Minor, E. y Aranda, R. (2017), "Determinantes económicos: evolución del costo de las calorías en México”, en J. A. Rivera, M. Hernández, C. A. Aguilar, F. Vadillo y C. Murayama (coords.), Obesidad en México. Recomendaciones para una politica de Estado, Ciudad de México, unam.

Mancero, X. (2001), "Estudios estadísticos y prospectivos. Escalas de equivalencia: reseña de conceptos y métodos", Estudios Estadísticos y Prospectivos de la CEPAL, núm. 8, Santiago de Chile, CePal.

Martínez, I. y Villezca, P. (2005), "La alimentación en México. Un estudio a partir de la Encuesta Nacional de Ingresos y Gastos de los Hogares y de las Hojas de Balance Alimenticio de la FAo", Ciencia UANL, vol. 8, núm. 2. Recuperado de <https:/www.redalyc.org/pdf/402/40280207.pdf>

Martínez-Puga, E. y Lendoiro, R. (2005), "Ingestas recomendadas de micronutrientes: vitaminas y minerales", en U. da Coruña, Fisiología y fisiopatología de la nutrición: I Curso de Especialización en Nutrición, Coruña, Universidade da Coruña. Recuperado de <http://ruc.udc.es/dspace/bitstream/2183/11338/1/CC-77 art 7.pdf>

Monteiro, C. A., Conde, W. L. y Popkin, B. M. (2001), "Independent effects of income and education on the risk of obesity in the brazilian adult population", The Journal of Nutrition, núm. 131, DOI <https://doi.org/10.1136/ adc.88.8.671>

Muñoz de Chávez, M., Ledesma, J., Chávez, A., Pérez, F., Méndoza, E., Calvo, C. y Melo, V. (2010), Composición de alimentos Miriam Muñoz de Chávez. Valor nutritivo de los alimentos de mayor consumo, México, McGraw-Hill. 
Nussbaum, M. (2011), “The central capabilities”, Creating Capabilities: The Human Development Approach, Massachusetts, Harvard University Press, DOI <https://doi.org/10.1080/13691457.2012.705970>

oms (2018), "Informe de la Nutrición Mundial 2018. Resumen Ejecutivo", Informe de la Nutrición Mundial 2018, Development Initiatives Poverty Research Ltd. Recuperado de <https://www.who.int/nutrition/globalnutritionreport/2018_Global_Nutrition_Report_Executive_Summary_ sp.pdf?ua $=1>$

Pérez, C. y Minor, E. (2011), "Cambio en los patrones de consumo alimentario en zonas rurales ante la crisis de precios de alimentos en México", en G. Felix-Verduzco y G. Aboites-Manrique (coords.), Dimensiones socioeconómicas de la pobreza en México, Saltillo, Universidad Autónoma de Coahuila.

Rivera, J., Campos-Nonato, I., Barquera, S. y González, T. (2017), "Epidemiología de la obesidad en México: magnitud, distribución, tendencias y factores de riesgo", en J. A. Rivera, M. Hernández, C. A. Aguilar, F. Vadillo y C. Murayama (coords.), Obesidad en México. Recomendaciones para una política de Estado, Ciudad de México, unam.

Rosado, J. L., Rivera, J., Lopez, G., Solano, L., Rodriguez, G., Casanueva, E. y Maulen, I. (1999), "Desarrollo y evaluación de suplementos alimenticios para el Programa de Educación, Salud y Alimentación”, Salud Pública de México, vol. 3, núm. 41, DOI <https://doi.org/10.1590/s003636341999000300003>

Schultz, T. (1961), "Investment in human capital", American Economic Review, vol. 1, núm. 51. Recuperado de $<$ http://la.utexas.edu/users/hcleaver 1330T/350kPEESchultzInvestmentHumanCapital.pdf>

(1962), "Reflections on investment in man", Journal of Political Economy, vol. 70, núm. 1. Recuperado de <https://econpapers.repec.org/ReP Ec:ucp:jpolec:v:70:y:1962:p:1>

Speckman, P. (1988), "Kernel smoothing in partial linear models", Journal of the Royal Statistical Society. Series B (Methodological), vol. 3, núm. 50. Recuperado de <http://www.jstor.org/stable/2345705>

Torres, F. (2017), “Transformación de la demanda alimentaria como factor de la obesidad en México", en J. A. Rivera, M. Hernández, C. A. Aguilar, F. Vadillo y C. Murayama (coords.), Obesidad en México. Recomendaciones para una politica de Estado, Ciudad de México, unam. 
